\title{
Light Curves of Type Ia Supernovae
}

\author{
A. V. Lyutykh ${ }^{1,2^{*}}$, M. V. Pruzhinskaya ${ }^{2,3}$, and S. I. Blinnikovev \\ ${ }^{1}$ Faculty of Physics, Moscow State University, Moscow, 119991 Russia \\ ${ }^{2}$ Sternberg Astronomical Institute, Moscow State University, \\ pr. Universitetskii 13, Moscow, 119234 Russia \\ ${ }^{3}$ Space Research Institute, Russian Academy of Sciences, ul. Profsoyuznaya 84/32, Moscow, 117997 Russia \\ ${ }^{4}$ Alikhanov Institute for Theoretical and Experimental Physics, National Research Center "Kurchatov \\ Institute," ul. Bol'shaya Cheremushkinskaya 25, Moscow, 117218 Russia \\ ${ }^{5}$ Dukhov All-Russian Research Institute of Automatics, ul. Sushchevskaya 22, Moscow, 127055 Russia \\ Received November 6, 2020; revised November 18, 2020; accepted November 26, 2020
}

\begin{abstract}
We have studied the light curves of type Ia supernovae (SNe Ia) and the physical parameters inferred from them. We have constructed both analytical and numerical light curves of SNe Ia. Using an empirical relation between the SN luminosity and light-curve parameters, we have managed to impose constraints on the hydrodynamic solutions obtained by the STELLA code and to produce a sample of models that describe the observational properties of real SNe maximally accurately. With this sample we have established a relationship between the opacity in SN Ia ejecta and the parameters being determined directly from observations. The method has been tested on two classical SNe Ia as an example: $2011 \mathrm{fe}$ and $2012 \mathrm{fr}$. The presented approach allows the opacity to be found without resorting to time-consuming computations.
\end{abstract}

DOI: $10.1134 / \mathrm{S} 1063773721010060$

Keywords: supernovae, light curves, Hubble-Lemaître parameter.

\section{INTRODUCTION}

Type Ia supernovae ( $\mathrm{SNe}$ Ia) are successfully used as cosmological distance indicators, because they have a high luminosity at maximum light (Betoule et al. 2014; Scolnic et al. 2018; Abbott et al. 2019). It is also important that this luminosity can be "standardized," i.e., reduced to the same value. An empirical relation between the luminosity of $\mathrm{SNe}$ at maximum light and the shape of their light curves was first published in Rust (1974) and Pskovskii (1977, 1984). With a small sample of SNe it was shown in these papers that the brightness of more luminous $\mathrm{SNe}$ Ia declines more slowly after maximum, i.e., the light curve looks broader. Later, it was found that the luminosity of SNe Ia also depends on their color (Hamuy et al. 1996; Tripp 1998). Several SN standardization methods and models have been developed to date, with the $\Delta m_{15}$ (Phillips 1993; Phillips et al. 1999), SALT2 (Guy et al. 2007), SNEMO (Saunders et al. 2007), and SUGAR (Léget et al. 2020) methods being among them.

There are several scenarios for the explosion of $\mathrm{SNe}$ Ia. Usually, this is the thermonuclear explosion

*E-mail: lutixalex1998@gmail.com of a $\mathrm{C}-\mathrm{O}$ white dwarf whose mass exceeded the Chandrasekhar one as a result of accretion (Schatzman's mechanism; Whelan and Iben 1973; Hachisu et al. 1996) or the merging of two white dwarfs with a total mass greater than the stability limit (Iben and Tutukov 1984; Webbink 1984). To explain the whole variety of observed SN Ia subtypes (91bg-like, Iax, 91T-like, 03fg-like, etc.), there are some alternative scenarios, such as the sub-Chandrasekhar scenario usually associated with weak explosions or the superChandrasekhar one for more luminous events (Polin et al. 2019; Hachisu et al. 2012; Fink et al. 2018; Hsiao et al. 2020). It is important to emphasize that it is not yet known which of the scenarios is realized in nature and if several of them work, then in what proportion.

SNe Ia made it possible to discover the accelerating expansion of the Universe (Riess et al. 1998; Perlmutter et al. 1999) or, more precisely, the necessity of introducing the lambda term or dark energy into cosmological models. This discovery was subsequently confirmed on the basis of WMAP (Wilkinson Microwave Anisotropy Probe, Spergel et al. 2003) and Planck (Planck Collaboration 2014, 2020) cosmic microwave background anisotropy 
measurements as well as baryon acoustic oscillations (Anderson et al. 2014). For more details on the current status of the theory and observations of the accelerating expansion of the Universe, see the review by Blinnikov and Dolgov (2019).

In recent years, a discrepancy between the HubbleLemaittre parameters deduced by various methods has been found (Verde et al. 2019). For example, the following Hubble-Lemaitre parameter is inferred by measuring the distances from $\mathrm{SNe}$ Ia (Riess et al. 2019):

$$
H_{0}=74.0 \pm 1.4 \mathrm{~km} \mathrm{~s}^{-1} \mathrm{Mpc}^{-1},
$$

while the Planck data (Planck Collaboration 2020) give

$$
H_{0}=67.4 \pm 0.5 \mathrm{~km} \mathrm{~s}^{-1} \mathrm{Mpc}^{-1} .
$$

Thus, the difference between the two distance scales is $\sim 10 \%$. To understand why the discrepancy in the values of the Hubble-Lemaitre parameter arises, it is necessary to thoroughly investigate the nature of the possible systematic errors in both approaches (Kowalski et al. 2008; Planck Collaboration 2016; Riess et al. 2016; Freedman et al. 2019).

The light curves are an important source of information about the SN progenitor star and the details of the explosion. There are various ways of describing the light curves of SNe Ia: the analytical approach (Arnett 1979), the semi-analytical approach (Sukhbold 2019), and through numerical simulations (STELLA, Blinnikov et al. 2019; LUCY, Lucy 2005; TARDIS, Kerzendorf and Sim 2014; SEDONA, Kasen et al. 2006; ARTIS, Kromer and Sim 2009). All these approaches, in some way or another, face the necessity of calibrating the light curves and require the certain assumptions about the pattern of radiation propagation through the $\mathrm{SN}$ ejecta. Moreover, it is not always possible to accurately determine the opacity that dramatically affects the light-curve shape.

In this paper we use observational properties of the SN Ia light curves, such as the Pskovskii-Phillips law, to find constraints on the hydrodynamic solutions obtained through STELLA simulations. As a result, using a limited sample of models that best describe real $\mathrm{SNe}$, we consider the relationship between the opacity of $\mathrm{SNe}$ and characteristic times on the light curve.

\section{DESCRIPTION OF THE MODELS AND COMPUTATION OF THE LIGHT CURVES BY THE STELLA CODE}

To compute the SN light curves with the STELLA hydrodynamic code (Blinnikov et al. 2006), we use the models by Woosley et al. (2007). These models are presented in Table 1.

The models differ between themselves in chemical composition. They all have the Chandrasekhar mass of $1.4 M_{\odot}$. The letter $m$ in the name reflects the fact that the chemical composition is slightly mixed, i.e., there is no complete homogenization of the star, the composition stratification is retained, but one layer passes into another not abruptly, but gradually. The numbers have the following meaning. The first two numbers denote the mass of radioactive ${ }^{56} \mathrm{Ni}$ immediately after the explosion in the central layers. For example, 02 means $0.2 M_{\odot}, 06$ means $0.6 M_{\odot}$, and so on (see Table 1). Then, the layers with nonradioactive iron-peak elements are designated in the same way. Next is the layer of intermediate elements ( silicon and sulfur). The remaining letters (mh) are a technical notation of the zoning used in the finite-difference scheme. For example, m040301mh has $0.4 M_{\odot}$ of radioactive ${ }^{56} \mathrm{Ni}$, then the $0.3 M_{\odot}$ iron-peak layer and the $0.1 M_{\odot}$ silicon-sulfur one. The rest, i.e., the outer layers, are the unburned original carbon and oxygen in equal fractions. Here, this is $0.6 M_{\odot}$. The kinetic energy of the initial model expansion closely corresponds to the caloric content of the $\mathrm{CO}$ mixture transition to the final composition minus the gravitational binding energy of the white dwarf before the explosion.

We directly compute the SN light curves based on these models using the STELLA radiation hydrodynamic code (Blinnikov et al. 2006). The STELLA code has been successfully applied to model SNe of various types, including SNe Ia (Blinnikov et al. 1998; Baklanov et al. 2005; Folatelli et al. 2006; Tominaga et al. 2009; Tsvetkov et al. 2012; Tauris et al. 2013; Volnova et al. 2017; Balakina et al. 2020). Woosley et al. (2007), Kromer and Sim (2009), Sim et al. (2010), Kozyreva et al. (2017), and Tsang et al. (2020) showed that STELLA agrees well with other known codes that are used to model the light curves of SNe.

\section{CHOOSING MODELS FOR OUR ANALYSIS}

In the next step of our work, we check whether the presented models follow the Pskovskii-Phillips empirical law:

$$
\begin{gathered}
M_{V}=-20.883(0.417) \\
+1.949(0.292) \Delta m_{15}(B),
\end{gathered}
$$

where $M_{V}$ is the absolute $V$ magnitude of the SN Ia and the parameter $\Delta m_{15}$ shows the total amount in magnitudes that the SN $B$ brightness declines within the first 15 days after maximum (Phillips 1993; Phillips et al. 1999). The results of our check are 
Table 1. Models of SNe Ia

\begin{tabular}{|c|c|c|c|c|c|}
\hline Model no. & Model name & $\begin{array}{l}\text { Mass of radioactive } \\
\text { nickel, } M_{56} \mathrm{Ni}\end{array}$ & $\begin{array}{l}\text { Agreement with } \\
\text { standardization } \\
\text { equation (4) }\end{array}$ & $E_{\text {kin }}($ foe $)$ & $\begin{array}{c}v_{\mathrm{CO}} \\
\text { (thousand } \\
\mathrm{km} \mathrm{s}^{-1} \text { ) }\end{array}$ \\
\hline 0 & $\mathrm{~m} 020209 \mathrm{mh}$ & 0.2 & no & 1.380 & 11.80 \\
\hline 1 & $\mathrm{~m} 020205 \mathrm{mh}$ & 0.2 & yes & 0.864 & 7.12 \\
\hline 2 & $\mathrm{~m} 010309 \mathrm{mh}$ & 0.1 & yes & 1.394 & 11.87 \\
\hline 3 & $\mathrm{~m} 050203 \mathrm{mh}$ & 0.5 & no & 1.104 & 8.02 \\
\hline 4 & $\mathrm{~m} 010305 \mathrm{mh}$ & 0.1 & yes & 0.880 & 6.10 \\
\hline 5 & $\mathrm{~m} 030307 \mathrm{mh}$ & 0.3 & no & 1.466 & 12.17 \\
\hline 6 & $\mathrm{~m} 060201 \mathrm{mh}$ & 0.6 & yes & 1.012 & 6.73 \\
\hline 7 & $\mathrm{~m} 070201 \mathrm{mh}$ & 0.7 & no & 1.176 & 8.31 \\
\hline 8 & $\mathrm{~m} 050205 \mathrm{mh}$ & 0.5 & no & 1.359 & 10.93 \\
\hline 9 & $\mathrm{~m} 040301 \mathrm{mh}$ & 0.4 & yes & 0.863 & 5.24 \\
\hline 10 & $\mathrm{~m} 020305 \mathrm{mh}$ & 0.2 & yes & 1.045 & 7.71 \\
\hline 11 & $\mathrm{~m} 070203 \mathrm{mh}$ & 0.7 & no & 1.435 & 11.30 \\
\hline 12 & $\mathrm{~m} 060203 \mathrm{mh}$ & 0.4 & no & 1.269 & 9.63 \\
\hline 13 & $\mathrm{~m} 040303 \mathrm{mh}$ & 0.4 & no & 1.117 & 8.07 \\
\hline 14 & $\mathrm{~m} 010209 \mathrm{mh}$ & 0.1 & yes & 1.214 & 10.32 \\
\hline 15 & $\mathrm{~m} 060205 \mathrm{mh}$ & 0.6 & no & 1.524 & 12.42 \\
\hline 16 & $\mathrm{~m} 070301 \mathrm{mh}$ & 0.7 & no & 1.354 & 9.97 \\
\hline 17 & $\mathrm{~m} 020303 \mathrm{mh}$ & 0.2 & yes & 0.787 & 4.95 \\
\hline 18 & $\mathrm{~m} 020207 \mathrm{mh}$ & 0.2 & yes & 1.122 & 8.94 \\
\hline 19 & $\mathrm{~m} 040305 \mathrm{mh}$ & 0.4 & no & 1.375 & 11.01 \\
\hline 20 & $\mathrm{~m} 050201 \mathrm{mh}$ & 0.5 & yes & 0.847 & 5.21 \\
\hline 21 & $\mathrm{~m} 080103 \mathrm{mh}$ & 0.8 & no & 1.419 & 11.23 \\
\hline 22 & $\mathrm{~m} 010307 \mathrm{mh}$ & 0.1 & yes & 1.135 & 8.99 \\
\hline
\end{tabular}

presented in Fig. 1. A specific model from Table 1 corresponds to each dot on the graph. As can be seen from the plot, not all of the presented models are consistent with the observational properties of SNe Ia.

However, in a present-day cosmological analysis more complex SN light-curve parametrization models are used for SN standardization. The most popular model is SALT2 (Guy et al. 2007). The main parameters of the SALT2 model are $x_{1}$ (stretch) and $c$ (color). The parameter $x_{1}$ characterizes the lightcurve shape, while the parameter $c$ is defined as the difference between the $(B-V)_{\max }$ color at maximum in the $B$ band and the mean $\langle B-V\rangle$. With these parameters the $\mathrm{SN}$ standardization equation looks as follows:

$$
M_{B}^{*}=M_{B}-\alpha x_{1}+\beta c,
$$

where $M_{B}^{*}$ is the absolute $B$ magnitude at maximum light, while $M_{B}, \alpha$, and $\beta$ are the parameters of the standardization equation.

A library in Python, SNCosmo, ${ }^{1}$ is used to

\footnotetext{
${ }^{1}$ https://sncosmo.readthedocs.io/en/latest/index.html.
} 


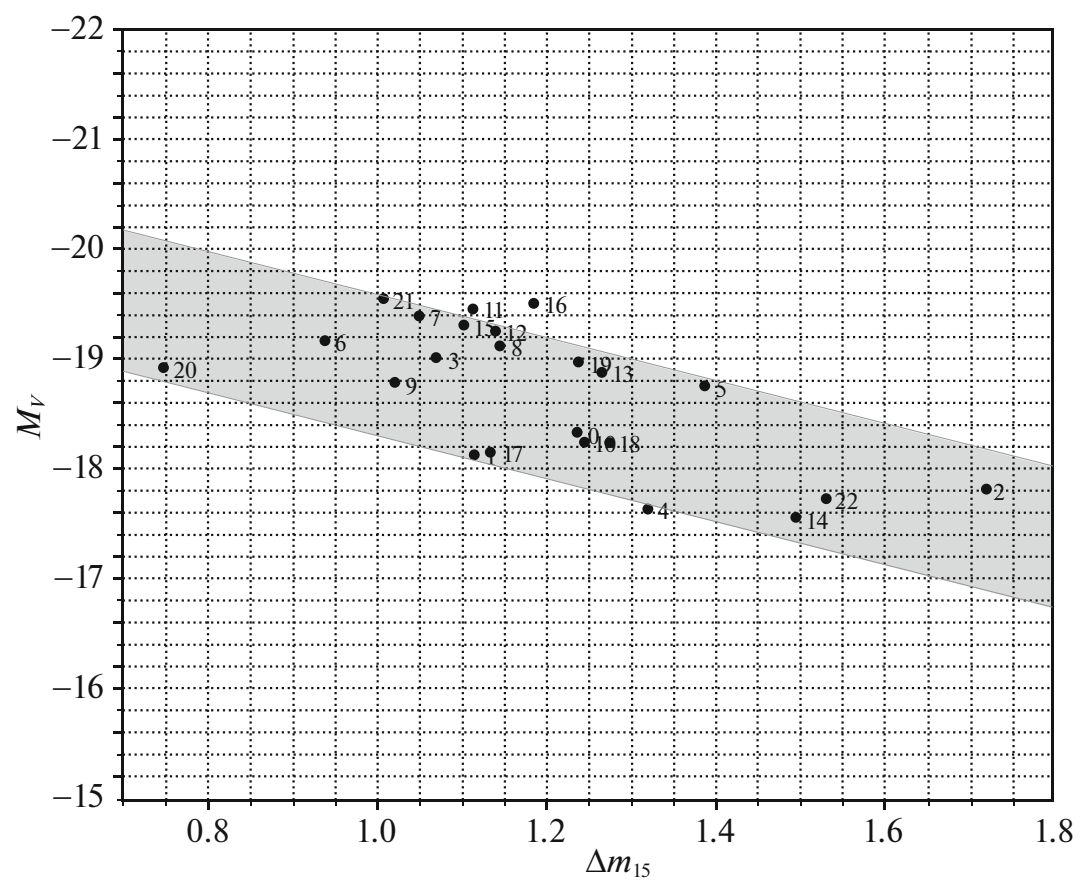

Fig. 1. Absolute $V$ magnitude at maximum light versus parameter $\Delta m_{15}$. The shaded region corresponds to the $1 \sigma$ scatter of $M_{V}$. The dots mark the models from Table 1 .

fit the light curves computed by STELLA with the SALT2 model. The fitting is done in three photometric bands: $B, V$, and $R$ (Bessell et al. 1990).

Using Eq. (4) and adopting the standardization parameters $M_{B}=-19.48, \alpha=0.154$, and $\beta=3.02$ (Scolnic et al. 2018), we will construct a twodimensional dependence of the absolute magnitude

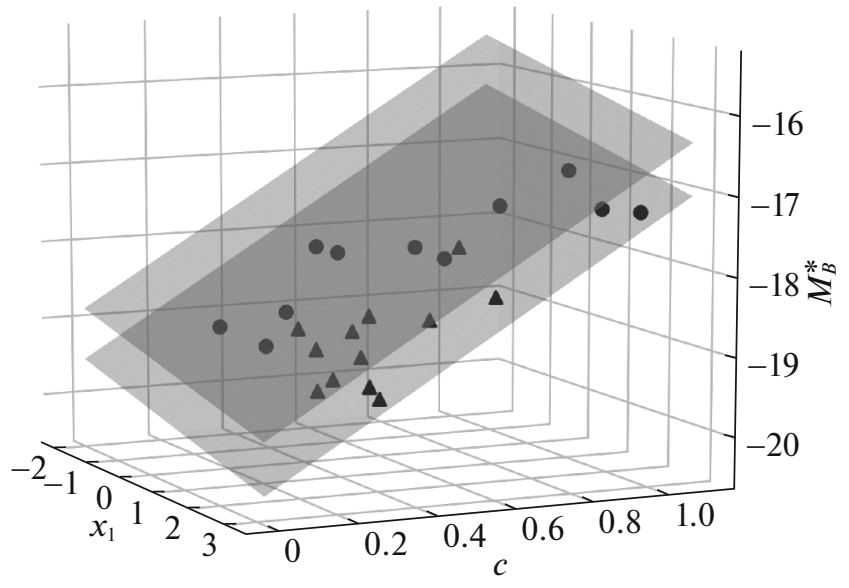

Fig. 2. Absolute $B$ magnitude at maximum light as a function of color parameter $(c)$ and light-curve shape parameter $\left(x_{1}\right)$. The planes limit the region of one standard deviation from the mean $M_{B}^{*}$. The dots mark the models satisfying the standardization equation (4) with the chosen parameters. The triangles mark the models that were not included in our sample.
$M_{B}^{*}$ on color parameter $c$ and light-curve shape parameter $x_{1}$ (Fig. 2). The planes limit the region of one standard deviation from the mean $M_{B}^{*}$. We will use the models falling into the $1 \sigma$ interval for our further analysis (see Table 1, column 4).

Let us check whether the selected models are consistent with the Pskovskii-Phillips relation (Fig. 3).

To summarize, it can be said that the light curves of the selected models satisfy the present-day standardization equation (Eq. (4), Scolnic et al. 2018) and the Pskovskii-Phillips relation (Eq. (3)) with a reasonable accuracy. As was shown in Woosley et al. (2007), the complete set of hundreds of models in no way satisfies the observed absolute magnitudelight-curve shape relationship, their natural selection occurs in nature. The details of such a selection have not yet been established by the theory. However, this does not prevent us from making useful predictions with our sample of SN models.

\section{DETERMINATION OF THE CHARACTERISTIC TIMES ON THE LIGHT CURVE}

In this section we discuss what dimensionless parameters can be inferred based on the analytical description of SN light curves.

For the models from Table 1 we will construct the bolometric light curves computed by the STELLA hydrodynamic code. In addition, using the analytical 


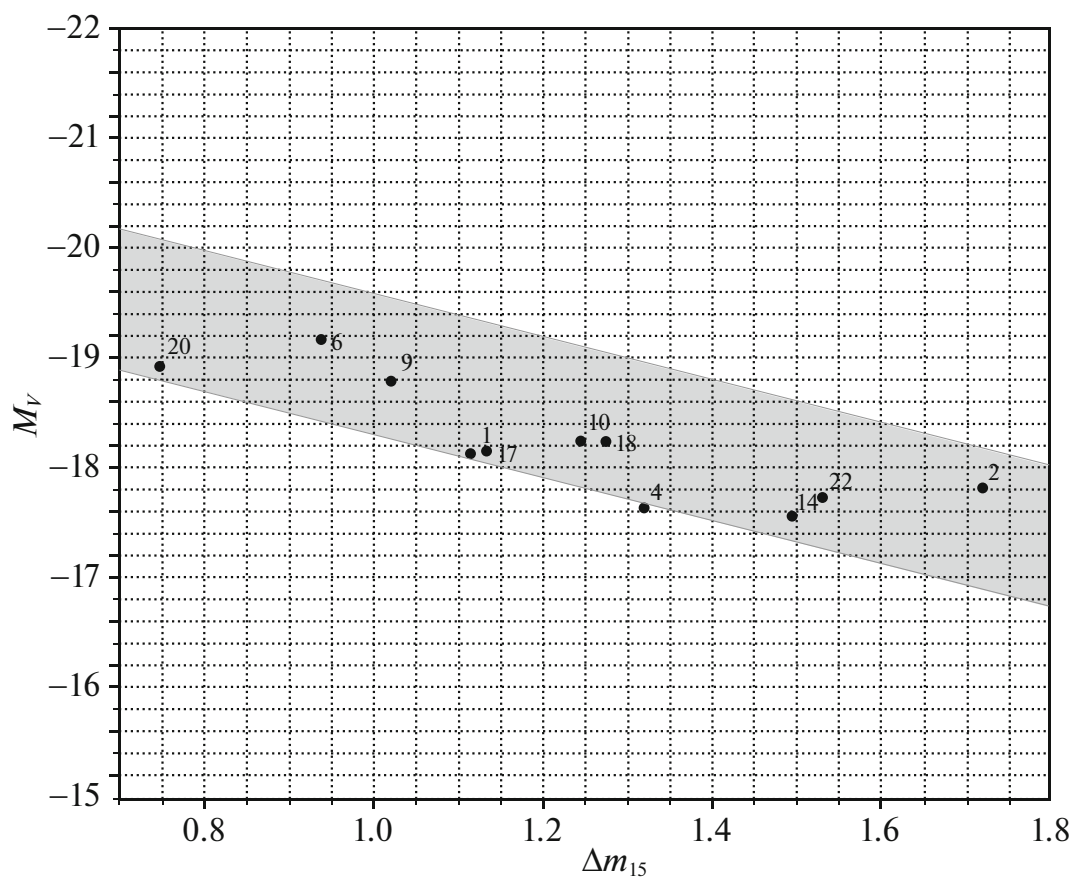

Fig. 3. Absolute $V$ magnitude at maximum light versus parameter $\Delta m_{15}$. The shaded region corresponds to the $1 \sigma$ scatter of $M_{V}$. The dots mark the models satisfying the standardization equation (4) with the chosen parameters; the numerical values correspond to the model number from Table 1.

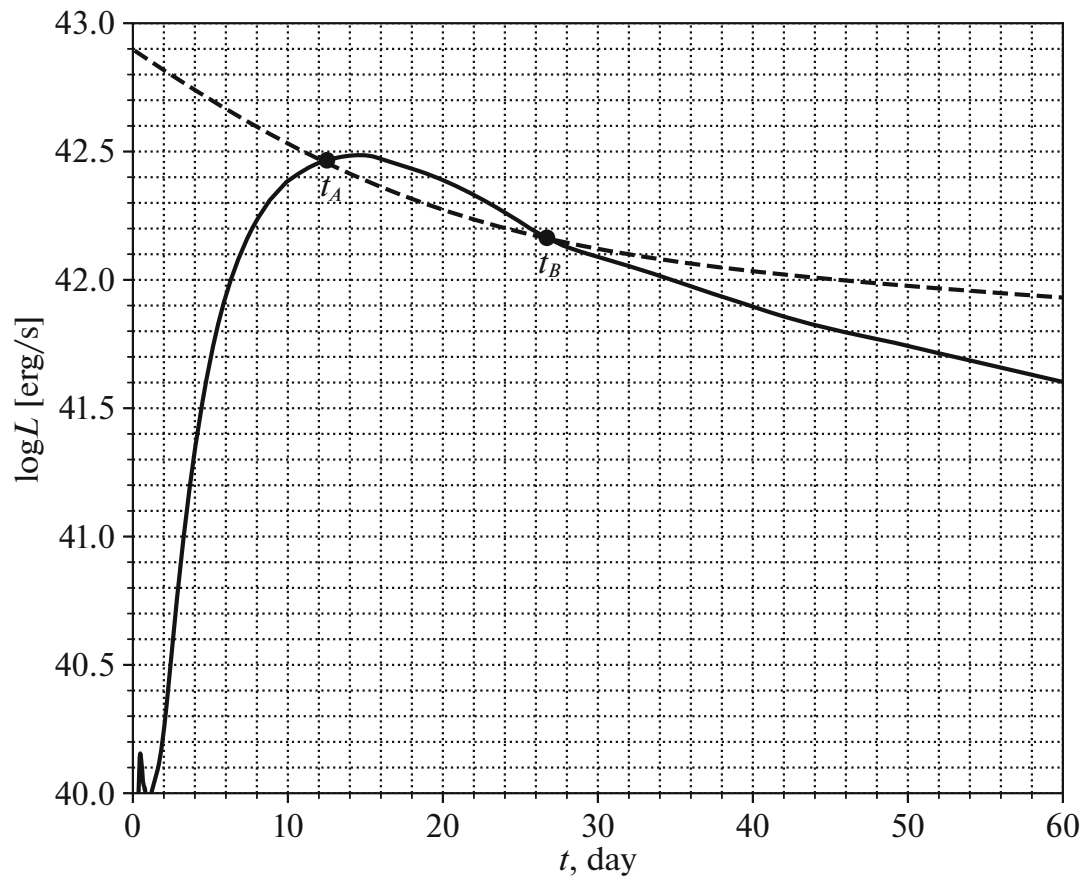

Fig. 4. The bolometric light curve $L_{\text {bol }}$ computed by STELLA and the power due to the gamma-ray deposition $L_{\gamma}$ (Arnett 1979) for the m020205mh model as an example. 


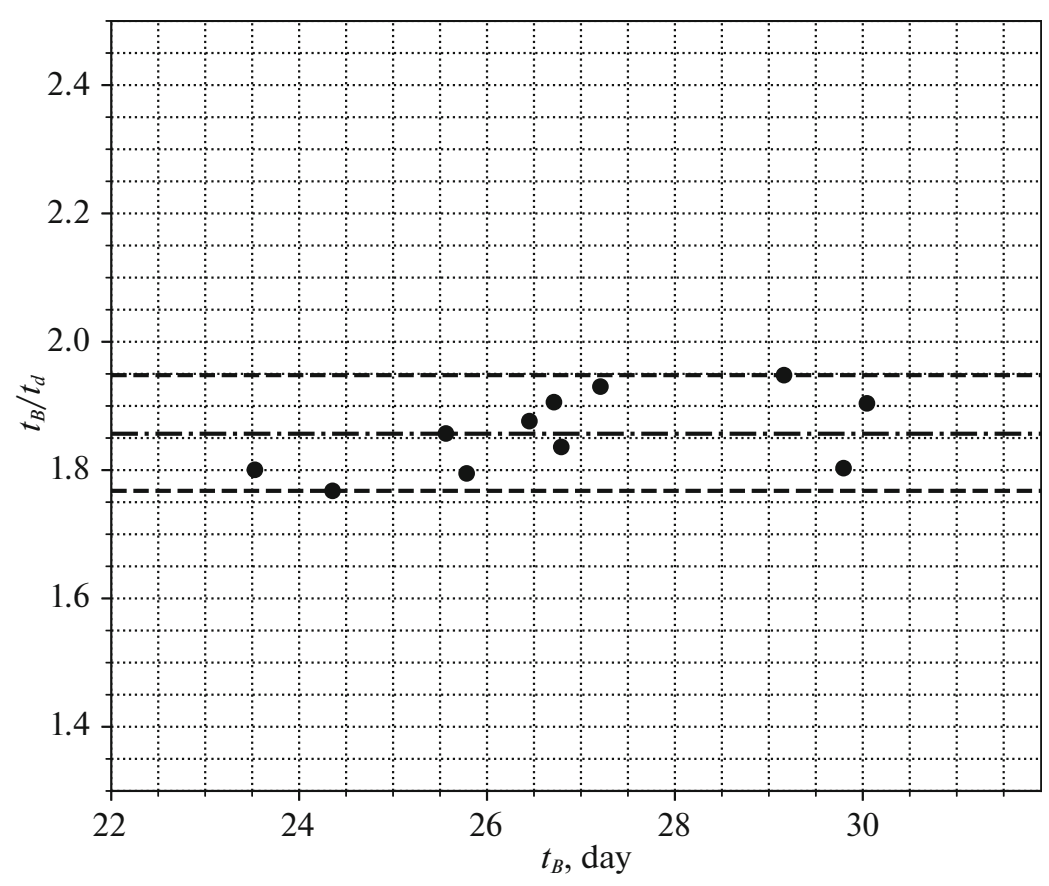

Fig. 5. The dependence of $t_{B} / t_{d}$ on $t_{B}$ calculated for our sample of models. The dashed lines bound the scatter of $t_{B} / t_{d}$, while the dash-dotted line corresponds to the mean $\left\langle t_{B} / t_{d}\right\rangle \simeq 1.86$.

description by Arnett (1979), we will compute the light curve due to the radioactive decay of nickel and cobalt $L_{\gamma}$ for each of them from the formula

$$
L_{\gamma}=\frac{M_{\mathrm{Ni}}}{M_{\odot}}\left(\epsilon_{\mathrm{Ni}} e^{-\frac{t}{t_{\mathrm{Ni}}}}+\epsilon_{\mathrm{Co}} e^{-\frac{t}{t_{\mathrm{Co}}}}\right),
$$

where $M_{\mathrm{Ni}}$ is the mass of the produced radioactive ${ }^{56} \mathrm{Ni}$ in fractions of the solar mass; $\epsilon_{\mathrm{Ni}}$ and $\epsilon_{\mathrm{Co}}$ are the specific energies being released during the radioactive decay of ${ }^{56} \mathrm{Ni}$ and ${ }^{56} \mathrm{Co}$, respectively; $t_{\mathrm{Ni}}$ and $t_{\mathrm{Co}}$ are the half-lives. The values of $\epsilon_{\mathrm{Ni}}, \epsilon_{\mathrm{Co}}, t_{\mathrm{Ni}}$, and $t_{\mathrm{Co}_{0}}$ are determined from nuclear data (see Table 2 ).

Let us construct the bolometric light curve $L_{\text {bol }}$ and the total radiated power $L_{\gamma}$ calculated from Eq. (5). The curves $L_{\text {bol }}$ and $L_{\gamma}$ intersect with each other at times $t_{A}$ and $t_{B}$ (Fig. 4).

Next, for the models under study let us consider the ratio $t_{B} / t_{d}$, where $t_{B}$ is the time of the second intersection of the bolometric light curve with the curve $L_{\gamma}$ and $t_{d}$ is the diffusion time defined as

$$
t_{d}=\sqrt{\frac{3 \kappa M_{e j}}{4 \pi c v}}
$$

where $M_{e j}$ is the ejecta mass, $v$ is the photospheric velocity, $\kappa$ is the opacity calculated with STELLA, $c$ is the speed of light (Arnett 1979). As can be seen from Fig. 5 , the values of $t_{B} / t_{d}$ for the sample of models under consideration lie in the interval $[1.77,1.95]$. The mean $\left\langle t_{B} / t_{d}\right\rangle$ is 1.86 , in agreement with $\sim 1.7$ found in Sukhbold (2019).
Similarly, for the sample of models we determine the time of the first intersection between the curves $L_{\text {bol }}$ and $L_{\gamma}\left(t_{A}\right)$ and construct the dependence of $t_{B} / t_{d}$ on $t_{A} / t_{d}$ (Fig. 6). As can be seen, the mean ratio $\left\langle t_{A} / t_{d}\right\rangle \simeq 0.99$, which would be expected, because the ejecta are heated on time scales of the order of the diffusion time.

In the next section, based on the derived ratio $t_{B} / t_{d}$, we will calculate the opacities for the models from our sample.

\section{OPACITY DETERMINATION}

In this section we obtain a constraint on the opacity $\kappa$ as a function of $t_{B}$. For this purpose, we use the dependence of $t_{B} / t_{d}$ on $t_{B}$ derived in the previous section (see Fig. 5) and the opacity-diffusion time relationship (Eq. (6)). Thus, we obtain the dependence shown in Fig. 7.

The mean opacity turns out to be $\langle\kappa\rangle \simeq 0.1$. Since $M_{e j}$ has the same numerical value for all models, the photospheric velocity $v$ has the greatest effect on the derived dependence. Since the opacity is difficult to determine without invoking resource-consuming computations, the presented approach can help in the primary estimation of its numerical value.

\section{COMPARISON WITH OBSERVATIONS}

In this section the above-described approach is used to determine the opacities of observed SNe Ia. 
Table 2. Nuclear data for ${ }^{56} \mathrm{Ni}$ and ${ }^{56} \mathrm{Co}$

\begin{tabular}{l|c|c}
\hline \multicolumn{1}{c|}{ Data } & ${ }^{56} \mathrm{Ni}$ & ${ }^{56} \mathrm{Co}$ \\
\hline Half-lives & $t_{\mathrm{Ni}}=8.8$ days & $t_{\mathrm{Co}}=111.3$ days \\
Released energy & $\epsilon_{\mathrm{Ni}}=6.45 \times 10^{43} \mathrm{erg} \mathrm{g}^{-1} \mathrm{~s}^{-1}$ & $\epsilon_{\mathrm{Co}}=1.45 \times 10^{43} \mathrm{erg} \mathrm{g}^{-1} \mathrm{~s}^{-1}$ \\
\hline
\end{tabular}

Table 3. Opacities and parameters of the best-fit STELLA models for SN $2011 \mathrm{fe}$ and SN $2012 \mathrm{fr}$

\begin{tabular}{c|c|c|c|c}
\hline SNe & Model & $t_{B}$, day & $M_{56 \mathrm{Ni}}, M_{\odot}$ & $\kappa, \mathrm{cm}^{2} \mathrm{~g}^{-1}$ \\
\hline SN 2011fe & m050201mh & $\sim 26.9$ & 0.5 & $0.10 \pm 0.01$ \\
SN 2012fr & m060201mh & $\sim 23.7$ & 0.6 & $0.08 \pm 0.01$ \\
\hline
\end{tabular}

As an example, we consider two SNe: SN 2011 fe and SN 2012fr. Both objects are classical SNe Ia and, hence, they satisfy the standardization equation. The bolometric light curves for SN $2011 \mathrm{fe}$ and SN 2012fr were taken from Dado and Dar (2015) and Contreras et al. (2018), respectively. Among the models presented in Table 1, we choose the best-fit model describing the observed light curve of each SN. The ${ }^{56} \mathrm{Ni}$ mass fraction to calculate the total radiated power in gamma rays using Eq. (5) is taken from the best-fit model parameters. The observations, the best-fit model, and $L_{\gamma}$ for SN 2011 fe and SN $2012 \mathrm{fr}$ are presented in Figs. 8 and 9, respectively.

Using the dependence of the opacity $\kappa$ on time $t_{B}$ presented in Fig. 7 , we determine the opacity. The value of $t_{B}$ is determined as the time of the second intersection between the bolometric model light curve and $L_{\gamma}$. The opacities found and the parameters of the best-fit STELLA models for SN $2011 \mathrm{fe}$ and SN $2012 \mathrm{fr}$ are presented in Table 3.

\section{DISCUSSION AND CONCLUSIONS}

In this section we discuss our results and their practical importance.

In the course of our study we computed the light curves of $\mathrm{SNe}$ Ia based on the models by Woosley et al. (2007) using the STELLA hydrodynamic code. From the constructed models we selected only those that satisfied the present-day standardization equation (Eq. (4), Scolnic et al. 2018) and the PskovskiiPhillips relation (Eq. (3)).

Using the selected models and Arnett's analytical description, we managed to constrain the ratio of the characteristic times $t_{A}$ and $t_{B}$ inferred from the SN light curves and diffusion time $t_{d}$. The mean ratio of the time $t_{B}$ to the diffusion time $t_{d}$ is consistent with the result presented in Sukhbold (2019). This ratio was used to determine the dependence of the opacity $\kappa$ on $t_{B}$.

The time $t_{B}$ is determined from the intersection of the bolometric light curve and the total radiated power in gamma rays $L_{\gamma}$. The value of $\kappa$ can then be determined by two methods. The first method uses the ratio $t_{B} / t_{d}$; the second method is based on a direct determination of the opacity from the dependence in Fig. 7. The second method is preferable, because the photospheric velocity entering into the definition of the diffusion time is a poorly determinable parameter. This approach was tested on real SNe: $2011 \mathrm{fe}$ and 2012fr. The theoretical models selected with the STELLA code for these SNe showed good agreement with observations. It is important to note that in the near future it will be possible to discover $\mathrm{SNe}$ within several days after the explosion (Jones et al. 2020). We will then be able to obtain detailed broadband photometry from the very beginning, which will improve significantly the accuracy of the observed bolometric light curves.

The models used were computed by assuming a spherically symmetric explosion of a $\mathrm{C}-\mathrm{O}$ white dwarf. However, for example, the DD explosion scenario suggests the existence of a large specific angular momentum, in contrast to the SD scenario. This, in turn, leads to an explosion asymmetry. The detection of a significant polarization of the optical continuum emission could be evidence for an explosion asymmetry and could serve as an independent argument for the scenario of merging white dwarfs. However, no continuum polarization in $\mathrm{SNe}$ Ia has been detected so far (Wang and Wheeler 2008), pointing to explosion sphericity or the existence of several 
LYUTYKH et al.

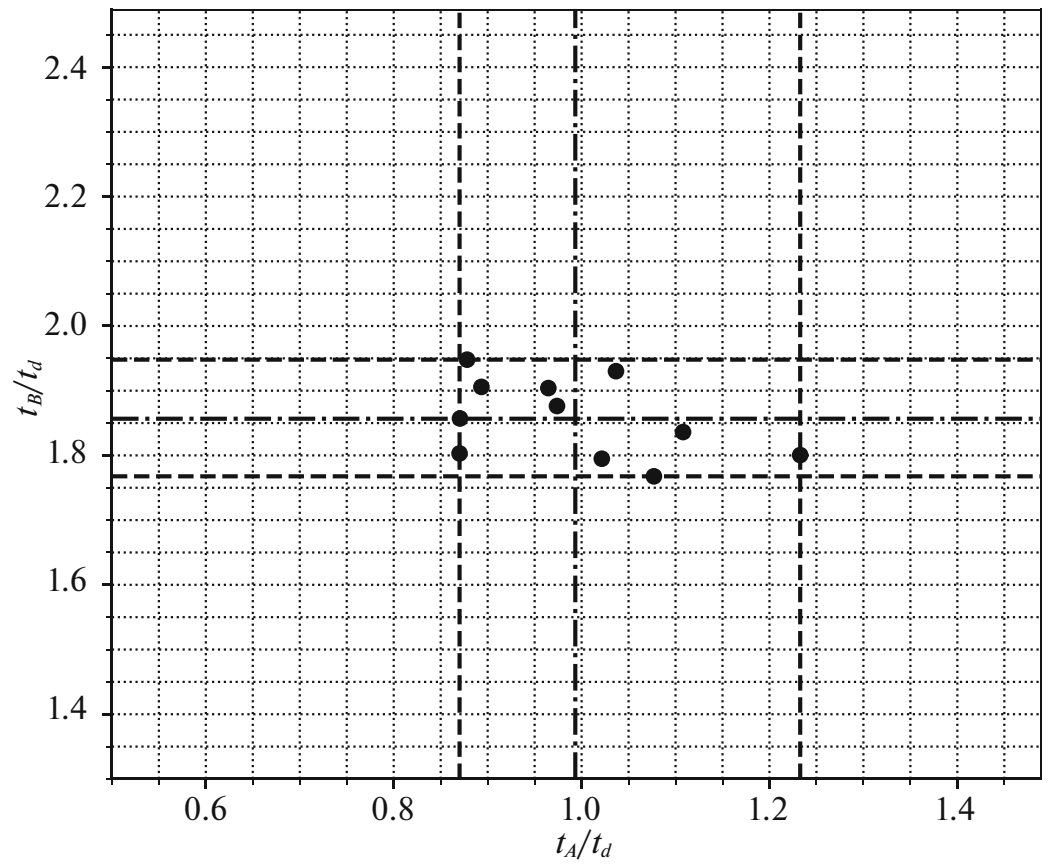

Fig. 6. The dependence of $t_{B} / t_{d}$ on $t_{A} / t_{d}$ calculated for our sample of models. The dashed lines bound the scatter of $t_{B} / t_{d}$ and $t_{A} / t_{d}$, while the dash-dotted lines correspond to the mean $\left\langle t_{B} / t_{d}\right\rangle \simeq 1.86$ and $\left\langle t_{A} / t_{d}\right\rangle \simeq 0.99$.

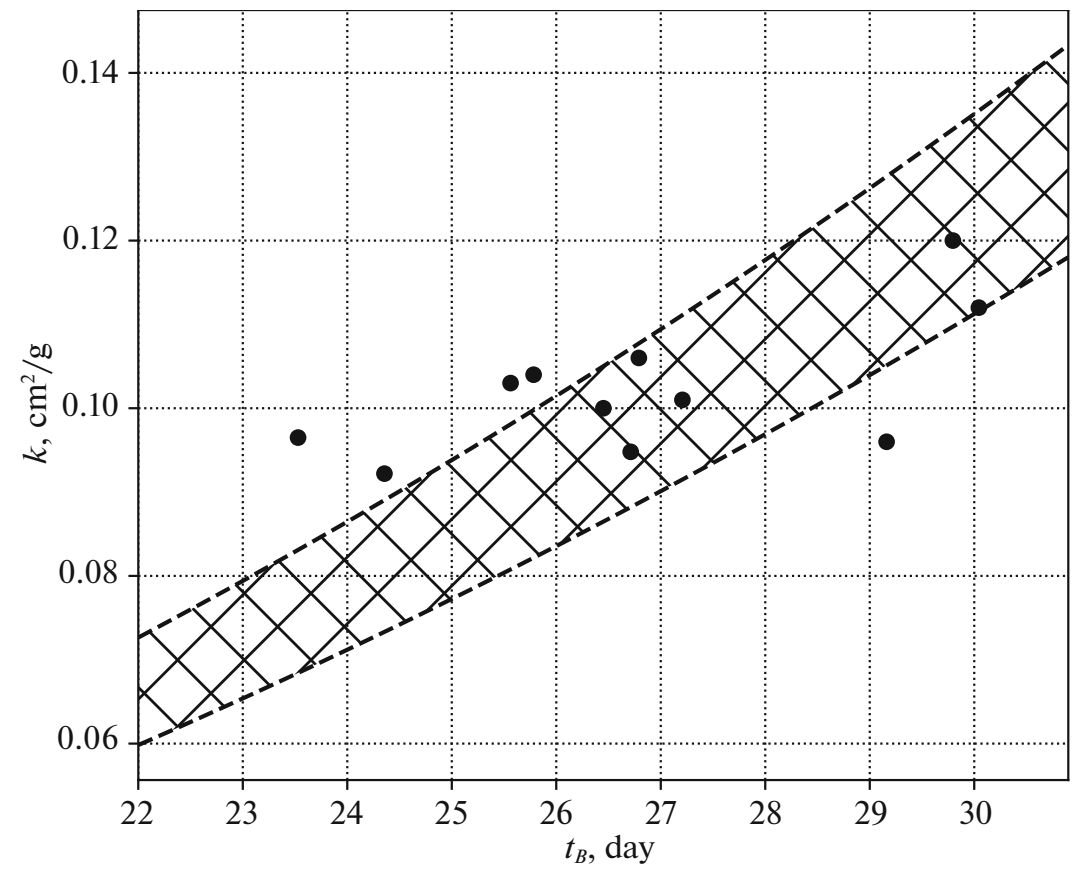

Fig. 7. Opacity $\kappa$ versus time $t_{B}$. The hatched region corresponds to the acceptable region of $t_{B} / t_{d}$ at the photospheric velocity $v=14000 \mathrm{~km} \mathrm{~s}^{-1}$. The dots mark the models from our sample.

SN Ia explosion scenarios and mechanisms (Cikota et al. 2019).

The dependence found allows the opacities for $\mathrm{SNe}$ Ia to be found without invoking time-consuming computations based on observations alone. Since we used the standardization equation whose parameters were calculated based on a large sample of real $\mathrm{SNe}$ 


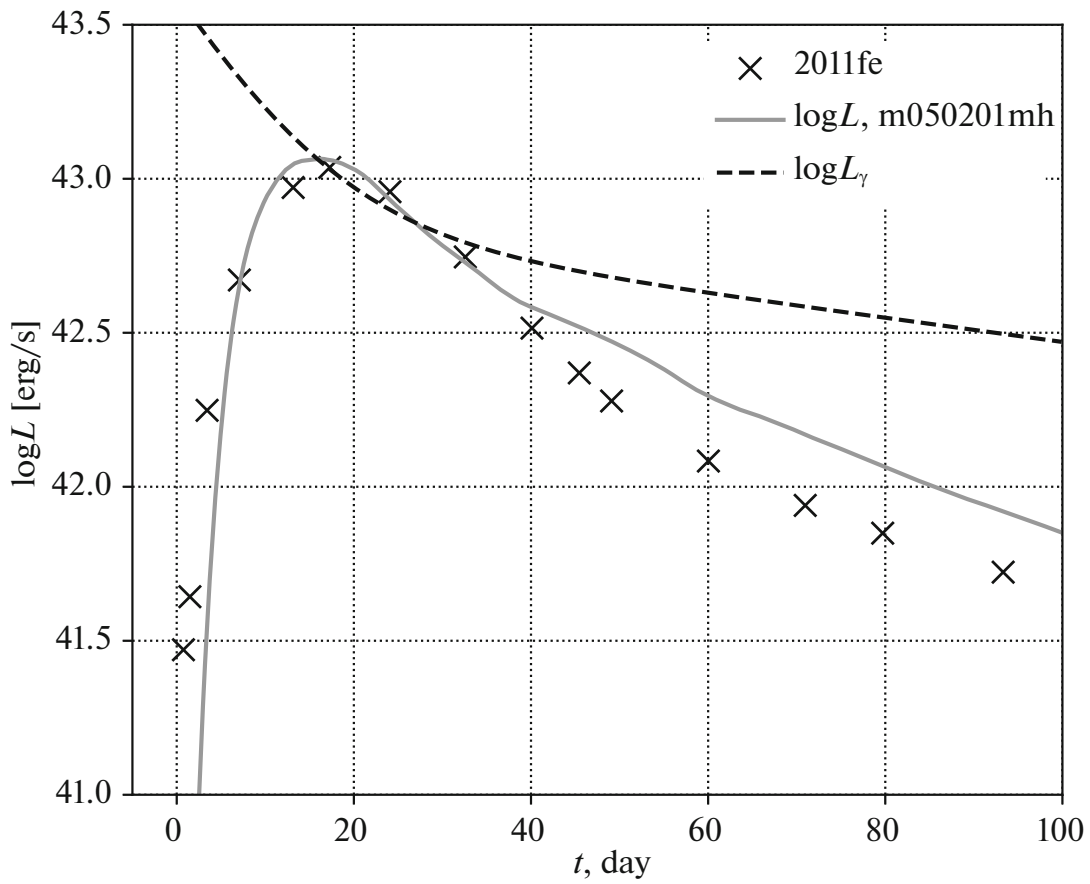

Fig. 8. The bolometric light curve (crosses; Dado and Dar 2015), the best-fit STELLA model (solid line), and the total radiated power in gamma rays $L_{\gamma}$ (dashed line) for SN 2011 fe.

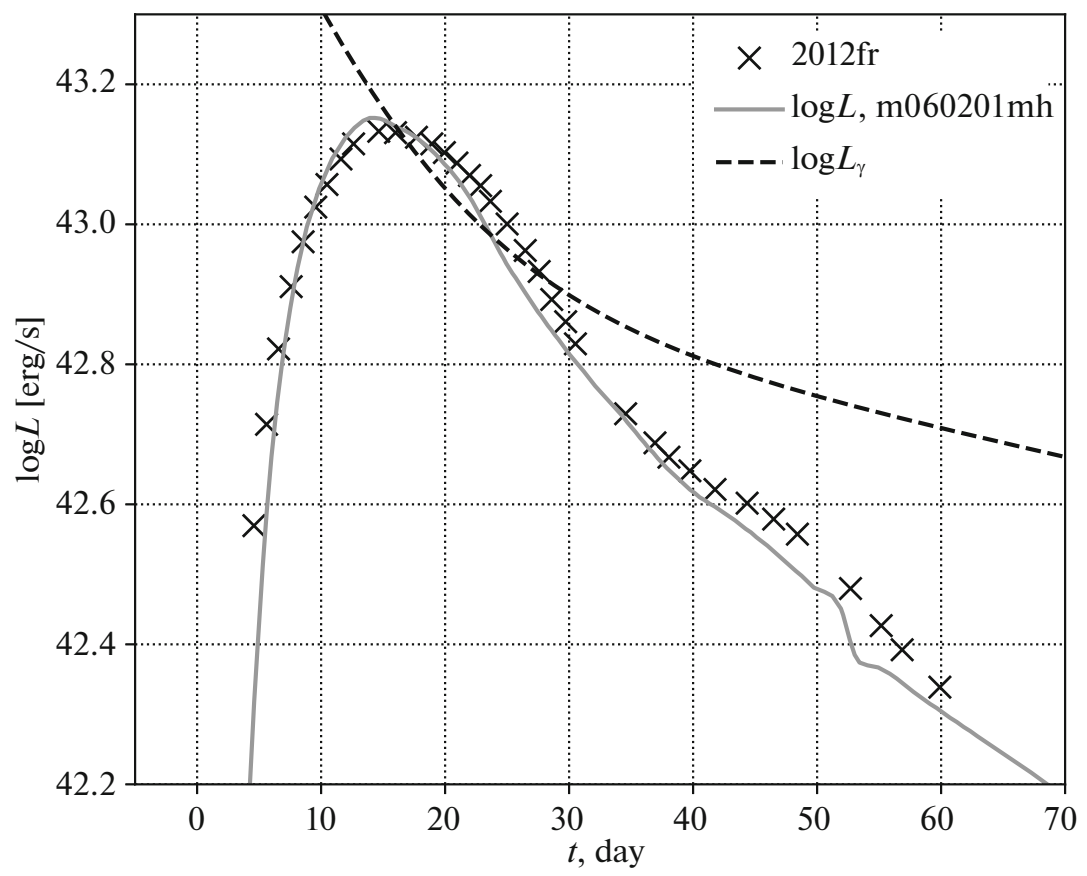

Fig. 9. The bolometric light curve (crosses; Contreras et al. 2018), the best-fit STELLA model (solid line), and the total radiated power in gamma rays $L_{\gamma}$ (dashed line) for SN $2012 \mathrm{fr}$.

for the selection of SN models, we expect the derived dependence to be applicable to most SNe Ia.

The goal of a future theory is to explain which SN Ia scenario and mechanism provide the pattern for the opacity found in this study from first principles.

\section{ACKNOWLEDGMENTS}

We are grateful to the anonymous referee for important remarks.

\section{FUNDING}

A.V. Lyukykh is grateful to the Russian Foundation for Basic Research and the Japan Society for the Promo- 
tion of Science within research project no. 19-52-50014 for their financial support in computing the light curves by the STELLA code. M.V. Pruzhinskaya thanks the Russian Science Foundation (project no. 18-12-00522) for its support in fitting the model light curves by the SNCosmo code. S.I. Blinnikov thanks the Russian Science Foundation (project no. 19-12-00229) for supporting the development and maintenance of the STELLA code.

\section{OPEN ACCESS}

This article is distributed under the terms of the Creative Commons Attribution 4.0 International Public License (http://creativecommons.org/licenses/by/4.0/), which permits unrestricted use, distribution, and reproduction in any medium provided you give appropriate credit to the original author(s) and the source, provide a link to the Creative Commons license, and indicate if changes were made.

\section{REFERENCES}

1. T. M. C. Abbott, S. Allam, P. Andersen, C. Angus, J. Asorey, A. Avelino, S. Avila, et al., Astrophys. J. 872, L30 (2019).

2. L. Anderson, E. Aubourg, S. Bailey, F. Beutler, V. Bhardwaj, M. Blanton, A. S. Bolton, J. Brinkmann, et al., Mon. Not. R. Astron. Soc. 441, 24 (2014).

3. W. D. Arnett, Astrophys. J. 230, L37 (1979).

4. W. D. Arnett, Ch. Fryer, and Th. Matheson, Astrophys. J. 33, 846 (2017).

5. P. V. Baklanov, S. I. Blinnikov, and N. N. Pavlyuk, Astron. Lett. 31, 429 (2005).

6. E. A. Balakina et al., arXiv e-prints 230, L37 (1979).

7. M. S. Bessell, Publ. Astron. Soc. Pacif. 102, 1181 (1990).

8. M. Betoule, R. Kessler, J. Guy, J. Mosher, D. Hardin, R. Biswas, P. Astier, P. El-Hage, et al., Astron. Astrophys. 568, A22 (2014).

9. S. I. Blinnikov and A. D. Dolgov, Phys. Usp. 62, 529 (2019)

10. S. I. Blinnikov and E. I. Sorokina, Astron. Astrophys. 356, L30 (2000).

11. S. I. Blinnikov, R. Eastman, O. S. Bartunov, V. A. Popolitov, and S. E. Woosley, Astrophys. J. 496, 454 (1998).

12. S. I. Blinnikov, F. K. Röpke, E. I. Sorokina, M. Gieseler, M. Reinecke, C. Travaglio, W. Hillebrandt, and $M$. Stritzinger, Astron. Astrophys. 453, 229 (2006)

13. N. N. Chugai, S. I. Blinnikov, R. J. Cumming, P. Lundqvist, A. Bragaglia, A. V. Filippenko, D. C. Leonard, Th. Matheson, and J. Sollerman, Mon. Not. R. Astron. Soc. 352, 1213 (2004).

14. A. Cikota, F. Patat, L. Wang, J. C. Wheeler, M. Bulla, D. Baade, P. H'oflich, S. Cikota, A. Clocchiatti, J. R. Maund, et al., Mon. Not. R. Astron. Soc. 490, $578(2019)$.

15. C. Contreras, M. M. Phillips, Ch. R. Burns, A. L. Piro, B. J. Shappee, M. D. Stritzinger, C. Baltay, P. J. Brown, et al., Astrophys. J. 859, 24 (2018).
16. S. Dado and A. Dar, Astrophys. J. 809, 32 (2015).

17. M. Fink, M. Kromer, W. Hillebrandt, F. K. Röpke, R. Pakmor, I. R. Seitenzahl, and S. A. Sim, Astron. Astrophys. 618, A124 (2018).

18. G. Folatelli, C. Contreras, M. M. Phillips, S. E. Woosley, S. Blinnikov, N. Morrell, N. B. Suntzeff, B. L. Lee, et al., Astrophys. J. 641, 1039 (2006).

19. W. L. Freedman, B. F. Madore, D. Hatt, T. J. Hoyt, In Sung Jang, R. L. Beaton, Ch. R. Burns, M. G. Lee, et al., Astrophys. J. 882, 34 (2019).

20. J. Guy, P. Astier, S. Baumont, D. Hardin, R. Pain, N. Regnault, S. Basa, R. G. Carlberg, et al., Astron. Astrophys. 466, 11 (2007).

21. I. Hachisu, M. Kato, and K. Nomoto, Astrophys. J. 470, L97 (1996).

22. I. Hachisu, M. Kato, H. Saio, and K. Nomoto, Astrophys. J. 744, 69 (2012).

23. M. Hamuy, M. M. Phillips, N. B. Suntzeff, R. A. Schommer, J. Maza, and R. Aviles, Astron. J. 112, 2391 (1996).

24. E. Y. Hsiao, P. Hoeflich, C. Ashall, J. Lu, C. Contreras, C. R. Burns, M. M. Phillips, L. Galbany, et al., Astrophys. J. 900, 140 (2020).

25. I. Iben, Jr. and A. V. Tutukov, Astrophys. J. Suppl. Ser. 54, 335 (1984).

26. D. O. Jones et al., Astrophys. J. Suppl. Ser. 54, 335 (1984).

27. D. Kasen, R. C. Thomas, and P. Nugent, Astrophys. J. 651, 366 (2006).

28. W. E. Kerzendorf and S. A. Sim, Mon. Not. R. Astron. Soc. 440, 387 (2014).

29. M. Kowalski, D. Rubin, G. Aldering, R. J. Agostinho, A. Amadon, R. Amanullah, C. Balland, et al., Astrophys. J. 686, 749 (2008).

30. A. Kozyreva, M. Gilmer, R. Hirschi, C. Frohlich, S. Blinnikov, R. T. Wollaeger, U. M. Noebauer, D. R. van Rossum, et al., Mon. Not. R. Astron. Soc. 464, 2854 (2017).

31. M. Kromer and S. A. Sim, Mon. Not. R. Astron. Soc. 398, 1809 (2009).

32. P. F. L'eget, E. Gangler, F. Mondon, G. Aldering, P. Antilogus, C. Aragon, S. Bailey, C. Baltay, et al., Astron. Astrophys. 636, A46 (2020).

33. L. B. Lucy, Astron. Astrophys. 429, 19 (2005).

34. S. Perlmutter, G. Aldering, G. Goldhaber, R. A. Knop, P. Nugent, P. G. Castro, S. Deustua, S. Fabbro, et al., Astrophys. J. 517, 565 (1999).

35. M. M. Phillips, Astrophys. J. 413, L105 (1993).

36. M. M. Phillips, P. Lira, N. B. Suntzeff, R. A. Schommer, M. Hamuy, and J. Maza, Astron. J. 118, 1766 (1999).

37. Planck Collab., Astron. J. 641, A1 (2020).

38. Planck Collab., Astron. J. 571, A16 (2014).

39. Planck Collab., Astron. J. 594, A1 (2016).

40. A. Polin, P. Nugent, and D. Kasen, Astrophys. J. 873, 84 (2019)

41. I. P. Pskovskii, Sov. Astron. 21, 675 (1977).

42. I. P. Pskovskii, Sov. Astron. 28, 658 (1984). 
43. A. G. Riess, A. V. Filippenko, P. Challis, A. Clocchiatti, A. Diercks, P. M. Garnavich, R. L. Gilliland, C. J. Hogan, et al., Astron. J. 116, 1009 (1998).

44. A. G. Riess, L. M. Macri, S. L. Hoffmann, D. Scolnic, S. Casertano, A. V. Filippenko, B. E. Tucker, M. J. Reid, et al., Astron. J. 826, 56 (2016).

45. A. G. Riess, S. Casertano, W. Yuan, L. M. Macri, and D. Scolnic, Astron. J. 876, 85 (2019).

46. B. W. Rust, The use of supernovae light curves for testing the expansion hypothesis and other cosmological relations (Univ. of Illinois, ORNL-4953, Ph.D. thesis, Oak Ridge National Lab., TN., 1974). B. W. Rust, PhD Thesis (1974b).

47. C. Saunders et al., Astrophys. J. 869, 167 (2018).

48. D. M. Scolnic, D. O. Jones, A. Rest, Y. C. Pan, R. Chornock, R. J. Foley, M. E. Huber, R. Kessler, et al., Astrophys. J. 859, 101 (2018).

49. S. A. Sim, M. Kromer, F. K. Röpke, E. I. Sorokina, S. I. Blinnikov, D. Kasen, and W. Hillebrandt, ASP Conf. Ser. 429, 148 (2010).

50. D. N. Spergel, L. Verde, H. V. Peiris, E. Komatsu, M. R. Nolta, C. L. Bennett, M. Halpern, G. Hinshaw, et al., Astrophys. J. Suppl. Ser. 148, 175 (2003).

51. T. Sukhbold, Astrophys. J. 874, 62 (2019).
52. T. M. Tauris, N. Langer, T. J. Moriya, Ph. Podsiadlowski, S. C. Yoon, and S. I. Blinnikov, Astrophys. J. 778, 23 (2013).

53. N. Tominaga et al., Astrophys. J. 705, L10 (2009).

54. R. Tripp, Astron. Astrophys. 331, 815 (1998).

55. B. T. H. Tsang, J. A. Goldberg, L. Bildsten, and D. Kasen, Astrophys. J. 898, 29 (2020).

56. D. Tsvetkov et al., Perem. Zvezdy 32, 6 (2012).

57. L. Verde, T. Treu, and A. G. Riess, Nat. Astron. 3, 891 (2019).

58. A. A. Volnova, M. V. Pruzhinskaya, A. S. Pozanenko, S. I. Blinnikov, P. Yu. Minaev, O. A. Burkhonov, A. M. Chernenko, Sh. A. Ehgamberdiev, et al., Mon. Not. R. Astron. Soc. 467, 3500 (2017).

59. L. Wang and J. C. Wheeler, Astron. J. 46, 433 (2008).

60. R. F. Webbink, Astrophys. J. 277, 355 (1984).

61. J. Whelan and I. Iben, Jr., Astrophys. J. 186, 1007 (1973).

62. S. E. Woosley, D. Kasen, S. Blinnikov, and E. Sorokina, Astrophys. J. 662, 487 (2007).

Translated by V. Astakhov 